\title{
Predictability of Bone Regeneration In Periodontal Surgery- A Review
}

\author{
Dr. Mahesh $\mathbf{J}^{1}$, Dr.Rethi Mahesh ${ }^{2}$, Dr.Jiji John ${ }^{3}$ \\ ${ }^{1}$ (Dept. of Periodontics, NICDS / Kerala University, India) \\ ${ }^{2}$ (Dept. of Conservative dentistry, NICDS / Kerala University, India) \\ ${ }^{3}$ (Dept. of Periodontics, CDSH,Rau / Indore University, India)
}

\begin{abstract}
Periodontal regeneration has become one of the primary objectives of periodontal therapy. Bone grafting has become a valuable mainstream clinical procedure in today's era of dentistry in variety of reconstructive applications. The periodontal regeneration in dentistry is producing better and expensive devices, although clinical measurements of attachment level and probing depths, along with radiography are good methods of evaluating tooth survival and prognosis. In addition, the regeneration industry may encourage the use of allograft and alloplasts which may serve as an implement to simple wound healing. This review is an assessment of the clinical use of various regenerative tools specifically bone replacements and membranes. The future of the regeneration may depend on the merging of various technologies and biological concepts. Including the possible use of biological barriers, various bone and periodontal growth inducers and artificial matrices that will attract or carry the cells necessary for regeneration
\end{abstract}

Keywords: Alveolar bone loss, Bone graft, Barrier membrane, periodontal, regeneration.

\section{Introduction}

Regeneration can be defined as the reproduction or reformation of organs or tissue that have been lost or injured as a result of a wound or infection. Regenerative periodontal procedure involves the creation of new alveolar bone, cementum, and periodontal ligament. Most periodontal practices focus on prevention of disease, initiation and corrective surgical treatment to eliminate deep pockets. Regeneration is distinct from tissue repair and is characterized by replacement of the damaged tissues with something that may be inferior to the original tissues both structurally and functionally. ${ }^{1}$

Eliminating bacteria and regenerating bone and supporting tissues helps in reducing pocket depth and repair damage caused by progression of periodontal disease. Over the last decade different modalities of regenerative treatment have been used and clinically applied. The positive effect of bone grafts and bone substitute on the outcome of periodontal regenerative procedures are well documented. At the present time, periodontists favor the bone as grafting material which has shown clinical effectiveness, functional periodontal repair, apparent bone defect fill and pocket reduction to manageable levels.

\section{Periodontal Debridement}

The removal of periodontal pathogenic bacteria, mineralized deposits on the root surface and infected cementum containing associated toxins is one of the most predictable methods achieved either by non surgical or surgical debridement leading to stable periodontal healing. ${ }^{2}$ Surgical debridement of intra-osseous defects appear to lead predictably to an increase in periodontal attachment of about $2.5 \mathrm{~mm}$ with variable amount of bone filling. ${ }^{3}$

\section{Terminology}

The definitions provided here are from The American Academy of Periodontontology and Glossary of Periodontic terms.

Regeneration is the reproduction or reconstitution of a lost or injured part, it means the formation of new bone, cementum and periodontal ligament on a previously diseased root surface.

Attachment apparatus refers to the cementum, the alveolar bone and the periodontal ligament.

New attachment means the union of connective tissue with a root surface that has been deprived of its periodontal ligament. This reunion occurs by the formation of new cementum with inserting collagen fibers.

Reattachment means the reunion of connective tissue with a root surface on which viable periodontal tissue is present.

Repair is healing of wound by tissue that does not fully restore the architecture or function of the part

Bone fill is the presence of hard tissue in a periodontal osseous defect

Intra-osseous defect (intra-bony) refers to a periodontal defect within bone 
Autograft is a tissue graft (bone) transferred from one position to a new position of the same individual. Allograft (homograft) is a tissue graft (bone) between individual of same species but with non identical genetic composition.

Xenograft (heterograft) is a tissue graft between members of different species ie animal to man.

Osteoconduction - Process in which the graft acts as a trellis or scaffold over which new host bone can form.

Osteoinduction - process in which new bone is induced to form through the action of factors contained within the grafted bone, such as proteins or growth factors.

\section{Historical Perspective}

In (1923) Hegdus attempted the use of bone grafts for the reconstruction of bone defects produced by periodontal disease ${ }^{5}$. Subsequent to their report and for the next several decades, the evolution of xenografts of various types became the main focus of attention. Buebe and Silvers $(1936)^{6}$ used boiled cow bone powder to successfully repair intra-bony defects in humans. Forceberg (1956) ${ }^{7}$ used Ox purum, in 11 human intra-bony defects. One showed excellent result and seven were satisfactory and rest unsatisfactory. Melcher $(1962)^{8}$ used anorganic bone in bovine bone in bone defects with a minimum follow-up of 3 years. He felt that protracted sequestration and slow resorption mitigated against the use of organic bone.

Scopp et al (1966), ${ }^{9}$ used Boplant bovine bone and reported pocket depth reduction at 6 months. Older $(1967)^{10}$ reported good results by measuring by probing depth reduction and increasing radiographic density. The widespread clinical use that followed these reports resulted in routine rejection and failure (Emmings $1974)^{11}$.The considerations that govern the selections of a material have been defined as ${ }^{12}$.

Biological acceptability

Predictability

Clinical feasibility

Minimal post operative sequelae

Patient acceptances

It is difficult to find a material with all these characteristics, and to date there is no ideal material or technique graft materials have been developed and tried in many forms given there limitations, experimental assessment of various bone grafting methods and other bone replacement materials have been undertaken with brief discussion of each is provided.

\section{Autogenous Bone Graft}

Autogenous grafts are those obtained from a remote location within the same host and are considered the gold standard bone replacement graft. These grafts are obtained intraorally from the extraction sockets, edentulous ridges, ramus, symphysis, tuberosity, or the surrounding buccal plate They are osteogenic, osteoconductive and osteoinductive. There is no risk of host rejection or disease transmission. But its major disadvantages are procurement morbidity, limited availability and high cost. There are several types of bone graft that have been or are being used clinically. They include cortical bone chips, osseous coagulum, bone blend, intraoral and extra oral cancellous bone, and marrow.

\subsection{Cortical bone chips}

The modern day use of periodontal bone grafts can be traced to the work of numbers and O' Heary $(1965)^{13}$. They reported that the use of cortical bone removed during osteoplasty and osteoctomy from sites when the surgical areas could use successfully to increase in bone height. According to (Langer et al 1986) suggests that this type of graft is still in use and may result in bone fill with decreased probing depth. From (Zayer and Yukna 1983) point of view the cortical chips due to their relative large particle size and potential for sequestration were replaced by osseous coagulum and bone blend.

\subsection{Osseous coagulum and bone blend}

The need of progenitors, blood supply and morphogens has encouraged the use of autogenous osteogenic tissue for grafting. For example osseous coagulum and bone blend has been and still is used to achieve bone filling in periodontal and osseous defects. ${ }^{16}$ The rationale for the use of their mixture as well as blood and osteogenic cells is to supply progenitors and morphogens to the wound site and pr5omote stable clot formation

\subsection{Iliac autografts}

The use of fresh and preserved iliac cancellous marrow bone has been extensively investigated. This material has been used by orthopedic surgeons for years data from human and animals studies support its use and the technique have prove successful in bone defects and in furcations ${ }^{17,18}$. However owing to problem 
associated with its use, such as postoperative infection, exfoliation, sequestration, root resorption and recurrence of the defect, it certain osteoclastic precursors that can promote root resorption and ankylosis. ${ }^{19}$

The advantages of using autogenous grafts are that these grafts are osteogenic, prevent disease transmission, and are cheap. However, they do require a second surgical site at the donor site. Schallhorn used iliac crest grafts in the treatment of infrabony defects and reported up to a 4-mm gain in bone height. However, reports of root resorption made this treatment option less favorable.

The fact that that intraoral autogenous grafts resulted in similar outcomes to bone obtained from extra oral sources made this a more favorable approach for the management of small defects. Vertical bone gains in infrabony defects average $3.5 \mathrm{~mm}$ for autogenous grafting materials, and several groups have shown that this approach may result in true periodontal regeneration with new cementum formation. ${ }^{25}$

\section{Bone Allografts}

It is nonvital, osseous tissue taken from one individual and transferred to another of the same species. They are osteoconductive and weakly osteoinductive. Its advantages include greater availability of banked bone than auto-graft and no additional surgical procedure needed. Its disadvantages include risk of disease transfer, not osteogenic, immunogenic, variable clinical results and expensive. The need for sufficient graft material, as well as the complications associated with second site surgery (used to obtain autogenous bone), led to the development of allograft and alloplastic graft materials, according to Urist and co- workers ${ }^{20}$ showed through numerous experiments that demineralization of a cortical bone graft induce new bone formation and greatly enhances its osteogenic potential. Demineralization with hydrochloric acid exposes the bone inductive proteins located in the bone matrix these proteins are collectively called bone morphogenetic protein (BMP), BMP stimulates the formation of new bone by osteoinductialized graft induce host cells to differentiate into osteoblast, whereas on undemineralized allograft is felt to function by osteoinduction as it affords a scaffold for new bone formation.

The rationale is that DMB is enriched with morphogens capable of inducing formation of mineralized connective tissues. ${ }^{21}$ Implication of demineralized bone matrix (DMB) into periodontal or bone defects can lead to increases in clinical attachment levels and increased bone filling. ${ }^{22}$ In this regard, the use of demineralized (freeze-dried bone allograft) or freeze-dried bone allograft (FDBA) has led to the filling of periodontal defects with mineralized bone-like material. However, both allograft and xenografts are foreign to the organism and therefore have the potential to provoke an immune response. Attempts have been made to suppress the antigenic potential of allograft \& xenograft by radiation, freezing and chemical treatment. Moreover bone allograft have been shown to persists as foreign and dried mineralized particles at the grafted site which might interfere with normal healing of bone. ${ }^{2}$

Studies on the clinical success of FDBA showed a bone fill ranging between $1.3-2.6 \mathrm{~mm}$ in periodontal defects. Using FDBA Mellonig found at least $50 \%$ bone fill in $67 \%$ of periodontal defects and this percentage increased to $78 \%$ when FDBA was combined with autogenous bone. Studies evaluating DFDBA reported a similar bone fill compared with FDBA, with an average range of 1.7-2.9 mm.Conversely, in a systematic review, fresh-frozen and DFDBA allografts were associated with significant improvements in bone level compared with open flap debridement, although this did not hold true for FDBA.

\section{Xenogenic Bone Grafts}

It is an osseous tissue that is harvested from one species, processed and then transferred to a recipient site of a different species. If properly prepared it is well tolerated by the tissues. It involves the risk of autoimmune disease. For oral applications, it generally comes in a granular or powder form, making it somewhat difficult to handle. It may also require some form of retentive structure, such as a membrane to hold the material in the desired location, because of an increased potential for migration of the material.

Even though positive clinical results have been reported for xenografts in the treatment of infrabony furcation, and endodontic-related surgical defects tissue/bone regeneration with xenografts might be unpredictable. One group evaluated the use of GTR by using anorganic bovine bone and a resorbable collagen membrane and after 1 year, $78 \%$ of defects healed successfully, although there were no differences in outcomes between GTR and no treatment of the residual periapical lesion. In contrast another group used anorganic bovine bone in combination with a bioabsorbable collagen membrane and found a success rate of $88 \%$ in the treatment group compared with $57 \%$ in the control group (no bone graft or membrane).

\section{Synthetic / Alloplastic Bone Grafts}

They are osteoconductive only. They eliminate the risk of disease transfer and procurement. An alloplast is a synthetic or inert foreign body that is implanted into host tissue. They are osteoconductive only and can be further categorized as hydroxyapatite, beta-tricalcium phosphate, non-ceramic, polymer, or bioactive glass . Alloplasts serve primarily to maintain space, and consequently they are not ideal for promoting 
periodontal regeneration. Hydroxyapatite grafts can achieve attachment gains of around 1-1.5 mm, and polymer grafts can average $2 \mathrm{~mm}$ of bone fill. However, tissue/bone regeneration is highly unpredictable in these cases .Alloplast materials are perhaps the most commonly investigated grafting materials for periapical defects. Positive results with respect to periodontal regeneration in periapical defects have been reported by using calcium sulfate, ceramic hydroxyapatite, and polylactide/polyglycolide copolymers

\section{Guided Tissue Regeneration And Cell Exclusion Techniques}

The principles of selective cell repopulation, ultimately termed GTR were developed by Nyman etal. ${ }^{25}$ by using selective approximation of periodontal instead of gingival tissue, new connective tissue attachment can form on previously diseased roots. The use of membrane filter also fulfilled the principles of tissues exclusion. Earlier studies had suggested exclusion of the oral epithelium could lead to improvements in periodontal healing after surgery. ${ }^{26}$

This concept lead to the development of epithelial exclusion methods, which apparently lead to turn to more predictable filling of intra-osseous defects around periodontally diseased teeth. ${ }^{27}$ in addition to bone grafting material in case of severe bone loss the use of barrier membrane in generative procedures may enhance clinical success by offering better protection and containment of bone substitute inside the defect.

The development of various barrier-based treatment modalities and techniques and a wide range of non resorbable and resorbable membrane gave rise to the acceptance of tissue regenerative approach focused solely on bone regeneration, guided bone regeneration. Histologic studies of bioabsorbable membranes indicate that regeneration can occur after healing, although repair was seen in certain cases. Bioabsorbable membranes have been found to achieve better regenerative outcomes in infrabony defects as compared with furcation

\section{Growth Factors/Cytokines/Host Modulating Agents}

The application of local growth factors has been studied to enhance the healing and regeneration potential of periodontal surgery. PRP, growth factors including BMPs, PDGF, and EMD are the most commonly used agents. Other promising therapeutics include collagen fragments bound to bone grafts, PTH, and transforming growth factor beta 3 (TGF-b3).

PRP is a highly concentrated suspension of autologous platelets, which secrete bioactive growth factors on activation. Because these growth factors are present at increased concentrations in PRP, they help to enhance key stages of wound healing and regenerative processes including chemotaxis, proliferation, differentiation, and angiogenesis. Similarly, autologous platelet concentrate (APC) contains PDGFs that promote regeneration. Studies comparing APC with a bioabsorbable membrane in infrabony defects found similar results between the 2groups, suggesting that APC could be used instead of a G T R membrane.

BMPs have an anabolic effect on periodontal tissues through stimulation of osteoblastic differentiation in human periodontal ligamentcell . Animal studies have shown new bone formation and connective tissue attachment with cementum regeneration occurred around circumferential periodontal defects in dogs treated with rhBMP-2compared with controls. However the use of BMPs in humans have not become widespread due to the finding of ankylosis in animal studies.. To date, sufficient human studies with BMPs in periodontal defects are lacking. PDGF is a growth factor involved in wound healing that stimulates the regenerative potential of periodontal tissues including bone, cementum, and periodontal ligament. PDGF-BB is one form of PDGF, and it has shown the most promise as a regenerative agent. PDGF has also been studied to enhance implant site development.

EMD are deposited onto the dentin root surface and provide the initial step in the formation of acellular cementum. Auto radiographic and scanning electron microscopy studies have provided additional evidence that EMD are responsible for both initiation and modulation of cementogenesis. Consequently, they have been incorporated into GTR attempts to promote cementogenesis and, thus, periodontal regeneration. Several studies have suggested that EMD and growth factors are promising in terms of their ability to promote tissue/bone regeneration, but that long-term data and sufficient evidence were still lacking.

A15 amino acid sequence from collagen has been incorporated into an inorganic bovine matrix and marketed as ABM/P-15 to rely on collagen to promote the binding of fibroblasts and osteoblasts in the tissue matrix. Here are only very few studies evaluating the ability of ABM/P-15 to promote periodontal regeneration. PTH is an endogenous hormone with anabolic actions in bone fused at low intermittent doses. Preliminary animal studies have shown that this might be a promising technique for regenerating bone in the periodontium.

Recently a arginine-glycine-aspartic acid (RGD) modified polyethylene glycol-based matrix (PEG) containing covalently boundpeptides of the parathyroid hormone (PTH(1-34)) to promote bone regeneration around dental implants was introduced. Studies found this technique enhanced bone regeneration in a similar magnitude as autogenous bone grafting in animals and more studies are needed. 


\section{Conclusion}

It is important to understand the various limitations in the assessment of periodontal regeneration, such as confirming the formation of bone rather than ectopically mineralized fibrous tissues, as well as the reformation of the attachment apparatus after therapy. The predictability of regeneration is affected by anatomic factors, as outline at the outset of this presentation and by the host systemic factors (eg. Smoking, chronic diseases) moreover as stated above, even with the best regenerative treatments available, it is probably appropriate every defect, so that simpler approaches to controlling diseases, which have greater evidences for long term success can be used. As our understanding of stem cells, matrix and morphogens increases, there is hope that their contribution to regeneration will eventually lead to combined therapy based on sound principles,

\section{References}

[1] Karring T, Nyman S et al. Development of the biological concept of guided tissue regeneration - animal and human studies; Periodontol 2000; 1: 26-35.

[2] Chan YK, Needleman IG et al, comparison of four methods of assessing root surface debridement. J Periodontol, 2000; 71(3): 385393.

[3] Polson AM, Heijl LC, Osseous repair in infrabony periodontal pockets. J Clin Periodontol 1978; 5(1): 13-23.

[4] Periodontal Regeneration: Bone grafts, James T. Melloning: Periodontal therapy. Vol. 1 1998, Quintessence publication co.

[5] Hegdus, Z.: The Rebuilding of the Alveolar Process by Bone Transplantation. Dent.

[6] Cosmos.65:736-742 (1923).

[7] Beube, F. E. and H. F. Silvers: Further Studies on Bone Regeneration with the Use of

[8] Boiled Heterogeneous Bone. J.PeriodontoL 7:17-21 (1936).

[9] Friedlander, G., M. Strong, and K. Sell: Studies on the Antigenicity of Bone. I. Freeze Dried and Deep Frozen Allografts in Rabbits. J. Bone Jt.Surg. 58A:854-858 (1976).

[10] Melcher, A. H.: The Use of Heterogeneous Anorganic Bone as an Implant Material in $\quad$ Oral Procedures. Oral Surg. 15:996-1000 (1962).

[11] Scopp, I. W., F. H. Morgan, J. J. Dooner, H. J. Fredrics, and R. A. Heyman: Bovine Bone (Boplant) Implants for Infrabony Oral Lesions. Periodontics4:169-176 (1966).

[12] Older, L. B.: The Use of Heterogeneous Bovine Bone Implants in the Treatment of Periodontal Pockets. J. Periodontol. 38:359-549 (1967).

[13] Autogenous and allogenic bone grafts in periodontal therapy, in routine rejection and failure (Emmings, 1974)

[14] Schallhorn RG : Present status of osseous grafting procedure; J Periodontol 1977; 48; 570

[15] Nabers, C. L. and T. J. O'Leary: Autogenous Bone Transplants in the treatment of Osseous Defects. J. Periodontol. 36:5-14 (1965).

[16] Langer, B., B. Wagengerg, and L. Langer: The use of frozen autogenous Bone in grafting procedures. Int. J.Periodontics Restorative Dent. 6(2):68-77 (1986).

[17] Zayner, D. J. and R. A. Yukna: Particle Size of Periodontal Bone Grafting Materials. J. Periodontol. 55:406-409 (1984).

[18] Froum SJ, Thaler R et al, Osseous autografts. Clinical responses to bone blend or hip

[19] marrow grafts; J Periodontol 1975;46(9) : 515-521.

[20] Schallhorn RG : the use of autogenous hip marrow biopsy implants for bony craters defects, J periodontal 1968;39: 145

[21] Schallhorn RG, Hiatt WH et al, iliac transplant in periodontal therapy, J Periodontol 1970; $41 ; 566$.

[22] Dragoo MR, Sullivan HC. A clinical and histological evaluation of autogenous iliac bone grafts in human. External root resorption. J Periodontol1973; 44(10) : 614-625.

[23] Urist MR , bone formation by auto induction. Science 1965; 150(698); 893- 899.

[24] Urist MR, bone formation by auto induction. Science 1965; 150(698); 893- 899.

[25] Mellonig JT , Bowers GM et al Clinical evaluation of freeze dried bone allografts in periodontal osseous defects. J Periodontol 1976: 47(3).

[26] Becker W, Clockie et al. histologic findings offer implantation and evaluation of different grafting materials and titanium microscrews into extraction sockets: Case report J. Periodontol 1998; 69 (4): 414-421

[27] Bowers G, Felton F, et al, histologic evaluation of regeneration in human intra-bony pockets when osteogenin is combined with demineralized freeze dried bone allograft and with purified bovine collagen. J. periodontal 1991; 62;690. 